MATHEMATICS OF COMPUTATION

Volume 73, Number 246, Pages 719-730

S $0025-5718(03) 01555-2$

Article electronically published on June 19, 2003

\title{
COMPUTATION OF MULTIPLE EIGENVALUES OF INFINITE TRIDIAGONAL MATRICES
}

\author{
YOSHINORI MIYAZAKI, NOBUYOSHI ASAI, YASUSHI KIKUCHI, DONGSHENG CAI, \\ AND YASUHIKO IKEBE
}

\begin{abstract}
In this paper, it is first given as a necessary and sufficient condition that infinite matrices of a certain type have double eigenvalues. The computation of such double eigenvalues is enabled by the Newton method of two variables. The three-term recurrence relations obtained from its eigenvalue problem (EVP) subsume the well-known relations of (A) the zeros of $J_{\nu}(z)$; (B) the zeros of $z J_{\nu}^{\prime}(z)+H J_{\nu}(z)$; (C) the EVP of the Mathieu differential equation; and (D) the EVP of the spheroidal wave equation. The results of experiments are shown for the three cases $(A)-(C)$ for the computation of their "double pairs".
\end{abstract}

\section{INTRODUCTION}

Two general theorems on the computation of eigenvalues of infinite matrices ( 7 and [6]) were proved by one of the co-authors (Ikebe). This has become a powerful tool for computing zeros of certain special functions as well as eigenvalues of certain differential equations. Such cases include:

(A) the computation of the zeros of $J_{\nu}(z)$ (where $J_{\nu}(z)$ denotes the Bessel function of the first kind of order $\nu)$ [6], [7],

(B) the computation of the zeros of $z J_{\nu}^{\prime}(z)+H J_{\nu}(z)$ [4,

(C) the eigenvalue problem (EVP) of the Mathieu differential equation [6], [8],

(D) the EVP of the spheroidal wave equation [9, and

(E) the computation of the zeros of regular Coulomb wave function and its first derivative 10 .

In this paper, let the two theorems be referred to as Theorem A and Theorem $\mathrm{B}$, respectively, for convenience:

Theorem A ([7 Theorems 1.1 and 1.4]). Assume a complex symmetric tridiagonal matrix

$$
\mathbf{A}=\left[\begin{array}{cccc}
d_{1} & f_{2} & & \mathbf{0} \\
f_{2} & d_{2} & f_{3} & \\
& f_{3} & d_{3} & \ddots \\
\mathbf{0} & & \ddots & \ddots
\end{array}\right]
$$

Received by the editor March 2, 2002 and, in revised form, August 12, 2002.

2000 Mathematics Subject Classification. Primary 34L16.

(C)2003 American Mathematical Society 
where $d_{k} \rightarrow 0, f_{k} \rightarrow 0(k \rightarrow \infty), f_{k} \neq 0(k=2,3, \ldots)$, representing a compact operator in the Hilbert space $\ell^{2}$. Let $\mathbf{A}$ have a simple eigenvalue $\lambda \neq 0$, and let $\mathbf{0} \neq \boldsymbol{\chi}=\left[\chi^{(1)}, \chi^{(2)}, \ldots\right]^{T} \in \ell^{2}$ be an eigenvector corresponding to $\lambda$. Then

(i) letting $\mathbf{A}_{n}(n=1,2, \ldots)$ denote the $n$th order principal submatrix of $\mathbf{A}$, there is a sequence $\left\{\lambda_{n}\right\}$ of eigenvalues of $\mathbf{A}_{n}$ which converge to $\lambda$;

(ii) letting $\chi^{T} \chi \neq 0$ and letting $\chi^{(n+1)} / \chi^{(n)}$ be bounded for all sufficiently large $n$, we have the following error estimate:

$$
\lambda-\lambda_{n}=\frac{f_{n+1} \chi^{(n)} \chi^{(n+1)}}{\chi^{T} \chi}[1+o(1)] \quad(n \rightarrow \infty) .
$$

Theorem B ([․, Theorem 1]). Assume a noncompact complex symmetric tridiagonal matrix

$$
\mathbf{T}=\left[\begin{array}{cccc}
d_{1} & f_{2} & & \mathbf{0} \\
f_{2} & d_{2} & f_{3} & \\
& f_{3} & d_{3} & \ddots \\
\mathbf{0} & & \ddots & \ddots
\end{array}\right]: D(\mathbf{T}) \rightarrow \ell^{2},
$$

where $0<\left|d_{k}\right| \rightarrow \infty(k \rightarrow \infty), 0<\left|f_{k}\right|<$ const $(k=2,3, \ldots), D(\mathbf{T})=$ $\left\{\left[u^{(1)}, u^{(2)}, \ldots\right]^{T}:\left[d_{1} u^{(1)}, d_{2} u^{(2)}, \ldots\right]^{T} \in \ell^{2}\right\}$. Let $\mathbf{T}$ have a simple eigenvalue $\lambda \neq 0$, let $\mathbf{0} \neq \chi=\left[\chi^{(1)}, \chi^{(2)}, \ldots\right]^{T}$ be an eigenvector corresponding to $\lambda$, and assume the existence of $\mathbf{T}^{-1}$. Then

(i) letting $\mathbf{T}_{n}(n=1,2, \ldots)$ denote the $n$th order principal submatrix of $\mathbf{T}$, there is a sequence $\left\{\lambda_{n}\right\}$ of eigenvalues of $\mathbf{T}_{n}$ which converge to $\lambda$;

(ii) letting $\chi^{T} \chi \neq 0$ and letting $f_{n+1} \chi^{(n+1)} / \chi^{(n)} \rightarrow 0(n \rightarrow \infty)$, we have the following error estimate:

$$
\lambda-\lambda_{n}=\frac{f_{n+1} \chi^{(n)} \chi^{(n+1)}}{\chi^{T} \chi}[1+o(1)] \quad(n \rightarrow \infty) .
$$

In Theorems $\mathrm{A}$ and $\mathrm{B}$, we define $\ell^{2}$ as the complex Hilbert space $\ell^{2} \equiv$ $\left\{\left[s_{1}, s_{2}, \ldots\right]^{T}: s_{1}, s_{2}, \ldots \in \mathbf{C}, \sum_{n=1}^{\infty}\left|s_{n}\right|^{2}<\infty\right\}$ and $o(1)$ as the quantity converging to zero as $n \rightarrow \infty$. The existence of $\mathbf{T}^{-1}$ is defined to have only one solution $\mathbf{z}=\mathbf{0}$ for $\mathbf{T z}=\mathbf{0}$. Also, we call an eigenvalue $\lambda$ simple if and only if its corresponding eigenvector is unique (up to scalar multiplication) and also if no corresponding generalized eigenvectors of rank 2 , namely, no vectors $\mathbf{0} \neq \mathbf{v}_{1} \in \ell^{2}$ (or $\mathbf{0} \neq \mathbf{v}_{2} \in D(\mathbf{T})$, in Theorem B's case) satisfying $(\mathbf{A}-\lambda \mathbf{I})^{2} \mathbf{v}_{1}=\mathbf{0}$ (or $(\mathbf{T}-\lambda \mathbf{I})^{2} \mathbf{v}_{2}=\mathbf{0}$, likewise) exist.

Theorems A and B justify an extremely accurate error estimates of eigenvalues for infinite matrices, but only when the eigenvalue is simple. Besides, it is known that the computation of double eigenvalues of matrices causes the severe drop of its precision. However, the authors came up with an algorithm to compute double eigenvalues accurately for some restricted matrix $\mathbf{T}$ in Theorem B.

The first aim of this article is to provide the theorems stating a necessary and sufficient condition that such (restricted) infinite matrices have double eigenvalues. This is discussed in Section 2. Furthermore, based on the theorems, the authors propose algorithms to compute double eigenvalues with a good rate of convergence and accuracy in Section 3 In fact, its computation is easily realized with the Newton method of two variables. Three examples ((A), (B), and (C)) are shown with the results of experiments (Section 4), followed by the concluding remarks in Section 5 


\section{Theorems on DOUble Eigenvalues}

2.1. Setting the type of infinite matrices. First, let the restricted matrix form of $\mathbf{T}$ in Theorem B be set as below:

[Matrix Form] Define the following infinite matrix T:

$$
\begin{aligned}
\mathbf{T} & =\left[\begin{array}{cccc}
d_{1} & f_{2} & & \mathbf{0} \\
f_{2} & d_{2} & f_{3} & \\
& f_{3} & d_{3} & \ddots \\
\mathbf{0} & & \ddots & \ddots
\end{array}\right]: D(\mathbf{T}) \rightarrow \ell^{2}, \\
D(\mathbf{T}) & =\left\{\left[v_{1}, v_{2}, \ldots\right]^{T}:\left[d_{1} v_{1}, d_{2} v_{2}, \ldots\right]^{T} \in \ell^{2}\right\},
\end{aligned}
$$

where $d_{n}=a_{n}+b_{n} \cdot \mu(n=1,2, \ldots), f_{n}=c_{n} \cdot \mu(n=2,3, \ldots)$, with $a_{n}, b_{n}$, and $c_{n}$ independent of $\mu \neq 0$, and in the forms of

$$
a_{n}=a n^{\alpha}[1+o(1)] \quad(n \rightarrow \infty), a \neq 0, \alpha>0 ; \quad\left|b_{n}\right| \leq B \quad(n=1,2, \ldots) ;
$$

and

$$
0 \neq c_{n}=C[1+o(1)](n \rightarrow \infty)(B \text { and } C \text { are constants })
$$

Letting $\lambda$ be an eigenvalue of $\mathbf{T}$ and letting $\mathbf{y} \equiv\left[y_{1}, y_{2}, \ldots\right]^{T}$ be the corresponding eigenvector, one could write

$$
\mathbf{T y}=\lambda \mathbf{y}, \quad \text { with } \mathbf{0} \neq \mathbf{y}=\left[y_{1}, y_{2}, \ldots\right]^{T} \in D(\mathbf{T}) \subset \ell^{2} .
$$

\subsection{Theorems on double eigenvalues with proofs.}

Theorem 1. If $\lambda$ is differentiable with respect to $\mu$, the following relation holds among $\lambda, \mu$, and $\mathbf{y}$ defined in (2.1) and (2.2):

$$
\left(\frac{\mathrm{d} \lambda}{\mathrm{d} \mu}\right) \cdot\left(\mathbf{y}^{T} \mathbf{y}\right)=\frac{1}{\mu} \cdot\left(\mathbf{x}^{T} \mathbf{x}\right)
$$

where

$$
\mathbf{0} \neq \mathbf{x} \equiv\left[x_{1}, x_{2}, \ldots\right]^{T}=\left[\sqrt{\lambda-a_{1}} \cdot y_{1}, \sqrt{\lambda-a_{2}} \cdot y_{2}, \ldots\right]^{T} \in \ell^{2}
$$

Proof. Let " " " denote the differentiation with respect to $\mu$. Then $(2.2)$ is changed into

$$
\lambda^{\prime} \mathbf{y}=(\mathbf{T}-\lambda \mathbf{I}) \mathbf{y}^{\prime}+\mathbf{T}^{\prime} \mathbf{y}
$$

Multiplying by $\mathbf{y}^{T}$ the left of both sides of this equation yields

$$
\left(\frac{\mathrm{d} \lambda}{\mathrm{d} \mu}\right) \mathbf{y}^{T} \mathbf{y}=\{(\mathbf{T}-\lambda \mathbf{I}) \mathbf{y}\}^{T} \mathbf{y}^{\prime}+\mathbf{y}^{T} \mathbf{T}^{\prime} \mathbf{y} .
$$

The first term vanishes if $\mathbf{y}^{\prime} \in \ell^{2}$. This is easily shown with $|\mathrm{d} \lambda / \mathrm{d} \mu|<\infty$ and (2.5). As for the second term of (2.6),

$$
\begin{aligned}
\mathbf{y}^{T} \mathbf{T}^{\prime} \mathbf{y} & =\mathbf{y}^{T}\left[\begin{array}{cccc}
d_{1}^{\prime} & f_{2}^{\prime} & & \mathbf{0} \\
f_{2}^{\prime} & d_{2}^{\prime} & f_{3}^{\prime} & \\
& f_{3}^{\prime} & d_{3}^{\prime} & \ddots \\
\mathbf{0} & & \ddots & \ddots
\end{array}\right] \mathbf{y}=\mathbf{y}^{T}\left[\begin{array}{cccc}
b_{1} & c_{2} & & \mathbf{0} \\
c_{2} & b_{2} & c_{3} & \\
& c_{3} & b_{3} & \ddots \\
\mathbf{0} & & \ddots & \ddots
\end{array}\right] \mathbf{y} \\
& =\mathbf{y}^{T} \cdot \frac{1}{\mu} \cdot \operatorname{diag}\left(\lambda-a_{1}, \lambda-a_{2}, \ldots\right) \mathbf{y}=\frac{1}{\mu} \cdot\left(\mathbf{x}^{T} \mathbf{x}\right) . \quad \square
\end{aligned}
$$


Remark 1. If $\mathbf{y}^{T} \mathbf{y} \neq 0$, it is obvious that $\mathrm{d} \lambda / \mathrm{d} \mu=0$ and $\mathbf{x}^{T} \mathbf{x}=0$ are equivalent. If $\mathbf{T}$ in (2.1) is real, this holds unconditionally since $\mathbf{y}$ is nonzero and real (or a real vector multiplied by a complex scalar).

Corollary 1. The following relation holds if $\mu$ is differentiable with respect to $\lambda$ :

$$
\left(\frac{\mathrm{d} \mu}{\mathrm{d} \lambda}\right) \cdot\left(\mathbf{x}^{T} \mathbf{x}\right)=\mu \cdot\left(\mathbf{y}^{T} \mathbf{y}\right)
$$

Proof. Refer to Theorem 1 for its proof.

Theorem 2. If $\mathbf{x}^{T} \mathbf{x} \neq 0$, the next three conditions are equivalent:

(a) $\lambda$ is a double eigenvalue of $\mathbf{T}$ in (2.1);

(b) $\mathrm{d} \mu / \mathrm{d} \lambda=0$;

(c) $\mathbf{y}^{T} \mathbf{y}=0$.

Proof. First, (b) $\Leftrightarrow(c)$ is obvious from (2.7). What remains is (a) $\Leftrightarrow(b)$. (a) $\Rightarrow(c)$ is shown first for proving $(\mathrm{a}) \Rightarrow(\mathrm{b})$. Since $\lambda$ is a double eigenvalue, there exists a generalized eigenvector $\mathbf{v} \in D(\mathbf{T})$ of rank 2 which satisfies

$$
(\mathbf{T}-\lambda \mathbf{I}) \mathbf{v} \equiv \mathbf{y} \neq \mathbf{0}, \quad \mathbf{y}, \mathbf{v} \in D(\mathbf{T}), \quad \text { and } \quad(\mathbf{T}-\lambda \mathbf{I})^{2} \mathbf{v}=(\mathbf{T}-\lambda \mathbf{I}) \mathbf{y}=\mathbf{0} .
$$

Now the computation of $\mathbf{y}^{T} \mathbf{y}$ leads to

$$
\begin{aligned}
\mathbf{y}^{T} \mathbf{y} & \left.=\{(\mathbf{T}-\lambda \mathbf{I}) \mathbf{v}\}^{T}(\mathbf{T}-\lambda \mathbf{I}) \mathbf{v}=\mathbf{v}^{T}(\mathbf{T}-\lambda \mathbf{I})^{2} \mathbf{v} \quad \text { (by the symmetry of } \mathbf{T}\right) \\
& =\mathbf{v}^{T} \mathbf{0} \quad\left(\text { by }(\mathbf{T}-\lambda \mathbf{I})^{2} \mathbf{v}=\mathbf{0}\right)=0 .
\end{aligned}
$$

The converse, or (b) $\Rightarrow$ (a) follows. Let " 1 " denote the differentiation with respect to $\lambda$. We will show the existence of $\mathbf{v} \in D(\mathbf{T})$ in (2.8). Differentiating (2.2) with respect to $\lambda$ gains

$$
\mathbf{T}^{\prime} \mathbf{y}-\mathbf{y}=-(\mathbf{T}-\lambda \mathbf{I}) \mathbf{y}^{\prime} .
$$

$\mathbf{T}^{\prime}=\mathbf{0}$ since $\mathrm{d} \mu / \mathrm{d} \lambda=0$ and $\mathbf{T}$ is dependent only on $\mu$. Therefore, $\mathbf{y}=(\mathbf{T}-\lambda \mathbf{I}) \mathbf{y}^{\prime}$. What needs to be shown is $\mathbf{0} \neq \mathbf{y}^{\prime} \in D(\mathbf{T})$. First, $\mathbf{y}^{\prime} \neq \mathbf{0}$ is easily shown, for, the assumption $\mathbf{y}^{\prime}=\mathbf{0}$ directly leads to $\mathbf{y}=\mathbf{0}$, which is absurd. Secondly, $\mathbf{y}^{\prime} \in D(\mathbf{T})$ is obvious again, since, otherwise, $\mathbf{y}=(\mathbf{T}-\lambda \mathbf{I}) \mathbf{y}^{\prime} \notin \ell^{2}$ is derived from the form of T.

\section{Computation of double pairs WITH THE NEWTON METHOD OF TWO VARIABLES}

In this section, we illustrate how to find the pairs $(\mu, \lambda)$ satisfying either (case 1) $\mathrm{d} \lambda / \mathrm{d} \mu=0$ or (case 2$) \mathrm{d} \mu / \mathrm{d} \lambda=0$. We use these results below for computing double eigenvalues of infinite matrices. Let such a pair $(\mu, \lambda)$ be called a double pair, and for a double pair of case 1 (case 2, respectively) let it be called a double pair of type $\mathrm{d} \lambda / \mathrm{d} \mu=0$ (a double pair of type $\mathrm{d} \mu / \mathrm{d} \lambda=0$, respectively).

3.1. Algorithm for computing double pairs of type $\mathbf{d} \lambda / \mathbf{d} \mu=0$. Now we are ready to propose the method for computing double pairs of type $\mathrm{d} \lambda / \mathrm{d} \mu=0$. Remark 1 states that if $\mathbf{y}^{T} \mathbf{y} \neq 0$, then double pairs of type $\mathrm{d} \lambda / \mathrm{d} \mu=0$ are equivalent to the $(\mu, \lambda)$ 's which satisfy $g_{1}(\mu, \lambda) \equiv \mathbf{x}^{T} \mathbf{x}=0$. Its algorithm is shown, by the Newton method of two variables. Throughout this section, $\lambda$ is assumed to be differentiable with respect to $\mu$. 
The Newton method of two variables, given $h_{1}(\mu, \lambda)$ and $h_{2}(\mu, \lambda)$, computes the pair $(\mu, \lambda)$ satisfying $h_{1}=h_{2}=0$ by iteration. Its formula is described as

$$
\left(\begin{array}{c}
\mu_{n+1} \\
\lambda_{n+1}
\end{array}\right)=\left(\begin{array}{c}
\mu_{n} \\
\lambda_{n}
\end{array}\right)-\mathbf{J}^{-1}\left(\mu_{n}, \lambda_{n}\right)\left(\begin{array}{c}
h_{1}\left(\mu_{n}, \lambda_{n}\right) \\
h_{2}\left(\mu_{n}, \lambda_{n}\right)
\end{array}\right),(n=0,1,2, \ldots),
$$

where

$$
\mathbf{J}\left(\mu_{n}, \lambda_{n}\right)=\left.\frac{\partial\left(h_{1}(\mu, \lambda), h_{2}(\mu, \lambda)\right)}{\partial(\mu, \lambda)}\right|_{\mu=\mu_{n}, \lambda=\lambda_{n}}=\left.\left(\begin{array}{cc}
\frac{\partial h_{1}}{\partial \mu} & \frac{\partial h_{1}}{\partial \lambda} \\
\frac{\partial h_{2}}{\partial \mu} & \frac{\partial h_{2}}{\partial \lambda}
\end{array}\right)\right|_{\mu=\mu_{n}, \lambda=\lambda_{n}} .
$$

$\mu_{0}, \lambda_{0}$ are initial values, and for $n \geq 1, \mu_{n}, \lambda_{n}$ are the approximated values of $\mu, \lambda$ after iteration of (3.1) $n$ times.

In order to apply this method to the problem in question, let us identify the terms that we want to have vanish. One is obviously $h_{1}(\mu, \lambda)=g_{1}(\mu, \lambda)=\mathbf{x}^{T} \mathbf{x}$, and the other is, in fact, $h_{2}(\mu, \lambda)=d_{1} y_{1}+f_{2} y_{2}-\lambda y_{1}$, the LHS of the first equation expanded from (2.2). The values required for each iteration are, from (3.2),

$$
\begin{aligned}
& \frac{\partial h_{1}}{\partial \mu}=2\left\{\left(\lambda-a_{1}\right) y_{1} \cdot \frac{\partial y_{1}}{\partial \mu}+\left(\lambda-a_{2}\right) y_{2} \cdot \frac{\partial y_{2}}{\partial \mu}+\cdots\right\}, \\
& \frac{\partial h_{1}}{\partial \lambda}=\left(\mathbf{y}^{T} \mathbf{y}\right)+2\left\{\left(\lambda-a_{1}\right) y_{1} \cdot \frac{\partial y_{1}}{\partial \lambda}+\left(\lambda-a_{2}\right) y_{2} \cdot \frac{\partial y_{2}}{\partial \lambda}+\cdots\right\}, \\
& \frac{\partial h_{2}}{\partial \mu}=b_{1} y_{1}+c_{2} y_{2}+\left(a_{1}+b_{1} \mu-\lambda\right) \cdot \frac{\partial y_{1}}{\partial \mu}+c_{2} \mu \cdot \frac{\partial y_{2}}{\partial \mu}, \\
& \frac{\partial h_{2}}{\partial \lambda}=\left(a_{1}+b_{1} \mu-\lambda\right) \cdot \frac{\partial y_{1}}{\partial \lambda}+c_{2} \mu \cdot \frac{\partial y_{2}}{\partial \lambda} .
\end{aligned}
$$

The values of $\partial y_{k} / \partial \mu, \partial y_{k} / \partial \lambda$ as well as $y_{k}(k=1,2, \ldots)$ appearing in (3.3)-(3.6) are calculated using three-term relations demonstrated below:

The expansion of (2.2) turns into the recurrence relations of $y_{k}$. By backward substitution, one can compute the values of $y_{k}$ from a sufficiently large $k=K$. Also, differentiating each of its expanded equations with respect to $\mu$ and $\lambda$ gives

$$
f_{k} \frac{\partial y_{k-1}}{\partial \mu}+d_{k} \frac{\partial y_{k}}{\partial \mu}+f_{k+1} \frac{\partial y_{k+1}}{\partial \mu}=\lambda \frac{\partial y_{k}}{\partial \mu}-\frac{\lambda-a_{k}}{\mu} y_{k}, \quad k=1,2, \ldots,
$$

and

$$
f_{k} \frac{\partial y_{k-1}}{\partial \lambda}+d_{k} \frac{\partial y_{k}}{\partial \lambda}+f_{k+1} \frac{\partial y_{k+1}}{\partial \lambda}=\lambda \frac{\partial y_{k}}{\partial \lambda}+y_{k}, \quad k=1,2, \ldots,
$$

respectively. Note that $\partial y_{0} / \partial \mu=\partial y_{0} / \partial \lambda \equiv 0$. With $y_{k}$ known, $\partial y_{k} / \partial \mu$ and $\partial y_{k} / \partial \lambda$ are obtained in the same way that the $y_{k}$ are computed.

Remark 2. In general, the use of the Newton method does not guarantee $\left(\mu_{k}, \lambda_{k}\right) \rightarrow$ $(\mu, \lambda)$ (a double pair). However, one can easily switch into applying the bisection method with the aid of (2.3). The bisection method ensures the convergence of approximated real $\left(\mu_{k}, \lambda_{k}\right)$ 's on the grounds that there exists the pair of 


$$
\begin{aligned}
& (\mu, \lambda)=\left(\tilde{\mu}_{1}, \tilde{\lambda}_{1}\right) \text { and }=\left(\tilde{\mu}_{2}, \tilde{\lambda}_{2}\right) \text { satisfying } \\
& \qquad\left.\left.(\mathrm{d} \lambda / \mathrm{d} \mu)\right|_{\mu=\tilde{\mu}_{1}, \lambda=\tilde{\lambda}_{1}} \cdot(\mathrm{d} \lambda / \mathrm{d} \mu)\right|_{\mu=\tilde{\mu}_{2}, \lambda=\tilde{\lambda}_{2}}<0 .
\end{aligned}
$$

From (2.3), the value of $\mathrm{d} \lambda / \mathrm{d} \mu$ is computable if only $\mathbf{y}^{T} \mathbf{y} \neq 0$. Equation (2.3) holds only for the $(\mu, \lambda)$ pairs satisfying (2.2), but its pairs can be obtained using the method in Theorem B.

3.2. Algorithm for computing double pairs of type $\mathrm{d} \mu / \mathrm{d} \lambda=0$. In this section, finding double pairs of another type is discussed. Throughout this section, it is assumed that $\mu$ is differentiable with respect to $\lambda$.

The same method that was used in Section 3.1 is applied. In this case, one of the terms that is to vanish is $h_{1}(\mu, \lambda) \equiv \mathbf{y}^{T} \mathbf{y}$, and the other is, as the last case, $h_{2}(\mu, \lambda)=d_{1} y_{1}+f_{2} y_{2}-\lambda y_{1}$. The values of $\partial h_{1} / \partial \mu, \partial h_{1} / \partial \lambda, \partial h_{2} / \partial \mu$, and $\partial h_{2} / \partial \lambda$ are derived by (3.5), (3.6), and

$$
\frac{\partial h_{1}}{\partial \mu}=2\left(y_{1} \cdot \frac{\partial y_{1}}{\partial \mu}+y_{2} \cdot \frac{\partial y_{2}}{\partial \mu}+\cdots\right), \quad \frac{\partial h_{1}}{\partial \lambda}=2\left(y_{1} \cdot \frac{\partial y_{1}}{\partial \lambda}+y_{2} \cdot \frac{\partial y_{2}}{\partial \lambda}+\cdots\right) .
$$

The values of $y_{k}, \partial y_{k} / \partial \mu$, and $\partial y_{k} / \partial \lambda$ are computed similarly to the computations in Section 3.1.

Remark 3. The bisection method is again applicable (useful especially when the Newton method does not work). Details are omitted (refer to Remark 2).

\section{Applications of double eigenvalue computation}

In this section we give three illustrations of the method in Section 3 . The first application is to show that there exist no complex zeros $z \neq 0$ of $J_{\nu}(z)$ satisfying $\mathrm{d} \nu / \mathrm{d} z=0$ (Section 4.1), the second is to compute zeros of $z J_{\nu}^{\prime}(z)+H J_{\nu}(z)$ satisfying $\mathrm{d} \nu / \mathrm{d} z=0$ (Section 4.2), and the third is to compute double eigenvalues of the Mathieu differential equations for both types $(\mathrm{d} \lambda / \mathrm{d} q=0$ and $\mathrm{d} q / \mathrm{d} \lambda=0$ ) (Section $4.3)$.

4.1. Proof of nonexistence of double pairs of $J_{\nu}(z)=0$. This section proves that there are no complex zeros $z \neq 0$ of $J_{\nu}(z)$ satisfying $\mathrm{d} \nu / \mathrm{d} z=0$.

$J_{\nu}(z)$, the Bessel function of the first kind of order $\nu$, is widely known to satisfy $J_{\nu}(z)-\{2(\nu+1) / z\} J_{\nu+1}(z)+J_{\nu+2}(z)=0$. Reference [6] proved that the problem of computing zeros $\nu$ of $J_{\nu}(z)$, given $z$ (the "inverse problem" of solving zeros $z$ of $J_{\nu}(z)$, so to speak) is equivalent to computing the eigenvalues of the following matrix:

$$
\mathbf{T}=\left[\begin{array}{cccc}
-1 & z / 2 & & \mathbf{0} \\
z / 2 & -2 & z / 2 & \\
& z / 2 & -3 & \ddots \\
\mathbf{0} & & \ddots & \ddots
\end{array}\right]: D(\mathbf{T}) \rightarrow \ell^{2}
$$

where

$$
D(\mathbf{T})=\left\{\left[w_{1}, w_{2}, \ldots\right]^{T}:\left[-1 w_{1},-2 w_{2}, \ldots\right]^{T} \in \ell^{2}\right\} .
$$

Figure 1 is the $z-\nu$ curve created by the algorithm in [6]. From this figure, it is conjectured that given $z \neq 0$, there are no $\nu$ satisfying $\mathrm{d} \nu / \mathrm{d} z \neq 0$. For $\nu>-1$, 


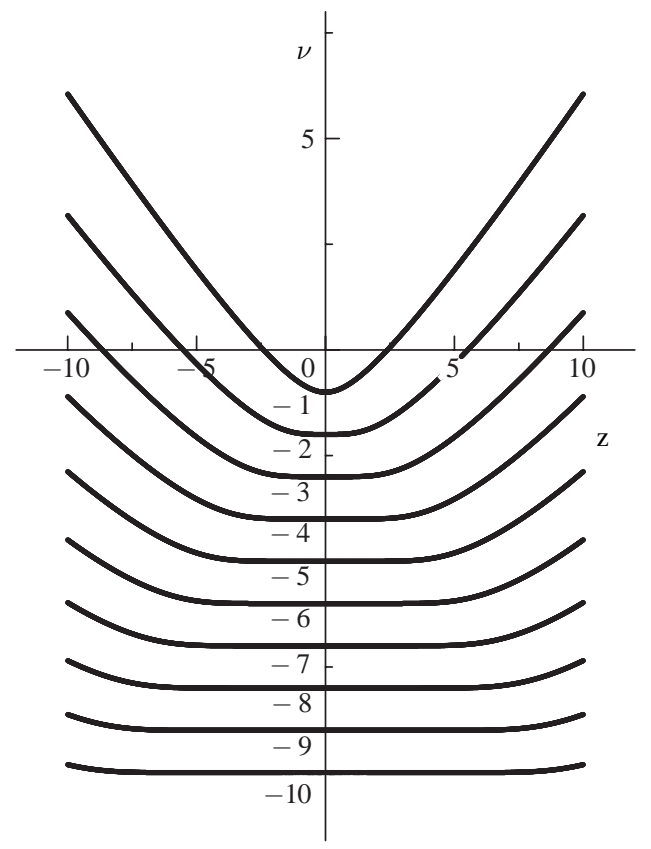

Figure 1. $(z, \nu)$ curves of $J_{\nu}(z)=0$

this is easily proved from the matrix theory. However, the proof demonstrated here covers the complex $z \neq 0$ and $\nu$.

One obtains the next relation when Theorem 1 is applied to this problem:

$$
\left(\frac{\mathrm{d} \nu}{\mathrm{d} z}\right)\left(\mathbf{y}^{T} \mathbf{y}\right)=\frac{1}{z}\left(\mathbf{x}^{T} \mathbf{x}\right)
$$

where

$$
\begin{aligned}
& \mathbf{y}=\left[J_{\nu+1}(z), J_{\nu+2}(z), \ldots\right]^{T} \in \ell^{2}, \\
& \mathbf{x}=\left[\sqrt{\nu+1} J_{\nu+1}(z), \sqrt{\nu+2} J_{\nu+2}(z), \ldots\right]^{T} \in \ell^{2} .
\end{aligned}
$$

Since $\mathbf{y} \in \ell^{2},\left|\mathbf{y}^{T} \mathbf{y}\right|<\infty$ is obvious. Then, it suffices to show $\mathbf{x}^{T} \mathbf{x} \neq 0$ to prove $\mathrm{d} \nu / \mathrm{d} z \neq 0$. Let $\mathbf{x}^{T} \mathbf{x}$ of (4.1) be computed as follows:

$$
\begin{aligned}
\mathbf{x}^{T} \mathbf{x}= & (\nu+1) J_{\nu+1}^{2}(z)+(\nu+2) J_{\nu+2}^{2}(z)+\cdots \\
= & \left\{(\nu+1) J_{\nu+1}^{2}(z)+(\nu+3) J_{\nu+3}^{2}(z)+\cdots\right\} \\
& +\left\{(\nu+2) J_{\nu+2}^{2}(z)+(\nu+4) J_{\nu+4}^{2}(z)+\cdots\right\} \\
= & \frac{z^{2}}{4}\left(J_{\nu}^{2}(z)-J_{\nu-1}(z) J_{\nu+1}(z)\right)+\frac{z^{2}}{4}\left(J_{\nu+1}^{2}(z)-J_{\nu}(z) J_{\nu+2}(z)\right) \\
= & \frac{z^{2}}{4} J_{\nu+1}(z)\left(J_{\nu+1}(z)-J_{\nu-1}(z)\right) \quad\left(\text { by } J_{\nu}(z)=0\right) \\
= & -z^{2} J_{\nu+1}(z) J_{\nu}^{\prime}(z) / 2 \quad\left(\text { since } 2 J_{\nu+1}^{\prime}=J_{\nu}-J_{\nu+2} \text { by [1] }\right) \\
\neq & 0 \quad\left(J_{\nu+1}(z) \neq 0 \text { and } J_{\nu}^{\prime}(z) \neq 0 \text { when } J_{\nu}(z)=0\right) .
\end{aligned}
$$

As was conjectured, no complex $z \neq 0$ and $\nu$ for $J_{\nu}(z)=0$ satisfy $\mathrm{d} \nu / \mathrm{d} z=0$. 


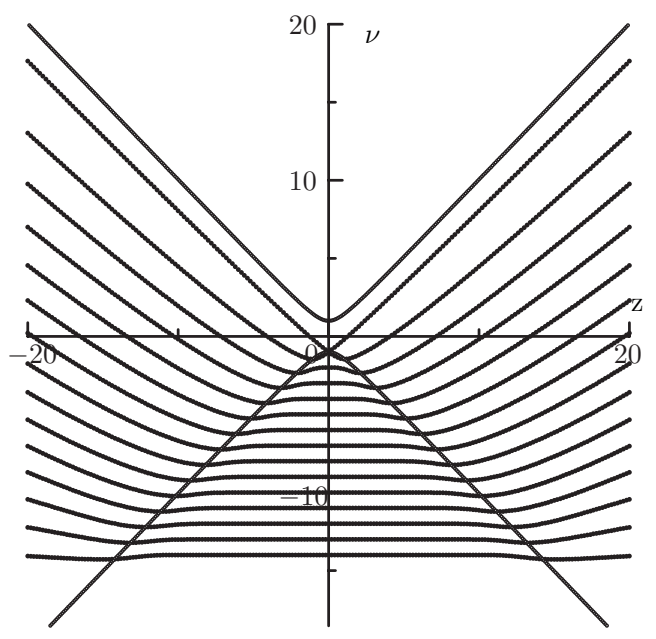

Figure 2. $(z, \nu)$ curves of $z J_{\nu}^{\prime}(z)+1 \cdot J_{\nu}(z)=0$ and $z^{2}-\nu^{2}+1=0$

4.2. Computation of double pairs of $z J_{\nu}^{\prime}(z)+H J_{\nu}(z)=0$. In [4], the distribution of the $(z, \nu)$ pair for $z J_{\nu}^{\prime}(z)+H J_{\nu}(z)=0$ was plotted (for $H=1$ ). What Figure 2 reveals in contrast with Figure 1 is that the existence of double pairs of type $\mathrm{d} \nu / \mathrm{d} z=0$ is observed. In fact, if we apply Theorem 1 to this problem, $\mathbf{x}^{T} \mathbf{x}=$ $(\nu+1) J_{\nu+1}^{2}+(\nu+3) J_{\nu+3}^{2}+(\nu+5) J_{\nu+5}^{2}+\cdots\left(\mathbf{x}=\left[\sqrt{\nu+1} J_{\nu+1}, \sqrt{\nu+3} J_{\nu+3}, \ldots\right]^{T}\right)=$ $\left(z^{2}-\nu^{2}+H^{2}\right) J_{\nu}^{2} / 4$ (this is derived similarly to Section 4.1 for $\mathbf{x}^{T} \mathbf{x}$; details are left

TABle 1. First 20 double pairs $(z, \nu)$ of type $\mathrm{d} \nu / \mathrm{d} z=0(H=1)$

\begin{tabular}{|c|c|c|}
\hline$N$ & $z$ & $\nu$ \\
\hline \hline 1 & $\pm 1.051328528 \cdots$ & $-1.450962327 \cdots$ \\
2 & $\pm 2.118898212 \cdots$ & $-2.343017207 \cdots$ \\
3 & $\pm 3.143427582 \cdots$ & $-3.298656842 \cdots$ \\
4 & $\pm 4.155369454 \cdots$ & $-4.274002258 \cdots$ \\
5 & $\pm 5.162139588 \cdots$ & $-5.258106611 \cdots$ \\
6 & $\pm 6.166349121 \cdots$ & $-6.246908153 \cdots$ \\
7 & $\pm 7.169132116 \cdots$ & $-7.238539584 \cdots$ \\
8 & $\pm 8.171052876 \cdots$ & $-8.232017074 \cdots$ \\
9 & $\pm 9.172420411 \cdots$ & $-9.226770626 \cdots$ \\
10 & $\pm 10.17341643 \cdots$ & $-10.22244598 \cdots$ \\
11 & $\pm 11.17415387 \cdots$ & $-11.21881075 \cdots$ \\
12 & $\pm 12.17470605 \cdots$ & $-12.21570577 \cdots$ \\
13 & $\pm 13.17512233 \cdots$ & $-13.21301815 \cdots$ \\
14 & $\pm 14.17543695 \cdots$ & $-14.21066546 \cdots$ \\
15 & $\pm 15.17567424 \cdots$ & $-15.20858602 \cdots$ \\
16 & $\pm 16.17585191 \cdots$ & $-16.20673270 \cdots$ \\
17 & $\pm 17.17598304 \cdots$ & $-17.20506883 \cdots$ \\
18 & $\pm 18.17607749 \cdots$ & $-18.20356539 \cdots$ \\
19 & $\pm 19.17614279 \cdots$ & $-19.20219915 \cdots$ \\
20 & $\pm 20.17618473 \cdots$ & $-20.20095122 \cdots$ \\
\hline
\end{tabular}


out) vanishes when $\mathrm{d} \nu / \mathrm{d} z=0$. Since $J_{\nu}(z) \neq 0$ (when $\left.z J_{\nu}^{\prime}(z)+H J_{\nu}(z)=0\right)$, $z^{2}-\nu^{2}+H^{2}=0$ should hold. This shows that double pairs exist, satisfying $z J_{\nu}^{\prime}(z)+H J_{\nu}(z)=0$ and $z^{2}-\nu^{2}+H^{2}=0$, which essentially agrees with [4] Theorem 4], stating that $z$ is a double root of $z J_{\nu}^{\prime}(z)+H J_{\nu}(z)=0$ if and only if $z^{2}-\nu^{2}+H^{2}=0$.

The algorithm in Section 3 was used for the computation of such pairs. The closest 20 points to the origin are shown with 10 significant digits in Table 1 . The $N$ 's represent that the corresponding points are the $N$ th closest to the origin (note that we have regarded two symmetrical points with respect to the $\nu$-axis as one).

\subsection{Computation of double eigenvalues of the Mathieu differential equa-} tion. The second-order linear differential equation

$$
w^{\prime \prime}(z)+(\lambda-2 q \cos 2 z) w(z)=0 \quad(0 \leq z<2 \pi),
$$

where $q$ and $\lambda$ are both parameters, is known as the Mathieu differential equation. Given $q$, the value of $\lambda$ which allows (4.2) to have a $\pi$ - or $2 \pi$-periodic solution $w(z)$ is called an eigenvalue, and such $w(z)$ is called the Mathieu function (of the first kind; as for the second kind, see [1]). The Mathieu function of the first kind is classified into four types, depending on the type of expansion ((Fourier) sine or cosine series expansion and the period $\pi$ or $2 \pi)$. In this section, only the type $w(z) \equiv s e_{2 m}(z, \lambda)=\sum_{k=1}^{\infty} B_{2 k} \sin (2 k) z, m=1,2,3, \ldots$, will be focused, since the other three types are essentially derived with the same procedure. The solutions for double pairs $(q, \lambda)$ of types $\mathrm{d} \lambda / \mathrm{d} q=0$ and $\mathrm{d} q / \mathrm{d} \lambda=0$ will be proposed in this section.

4.3.1. Double pairs of type $\mathrm{d} \lambda / \mathrm{d} \mu=0$. We shall first plot, in Figure 3 , the real pairs of $q$ and eigenvalues $\lambda$ of (4.2) [8]. Figure 3 suggests that there are points

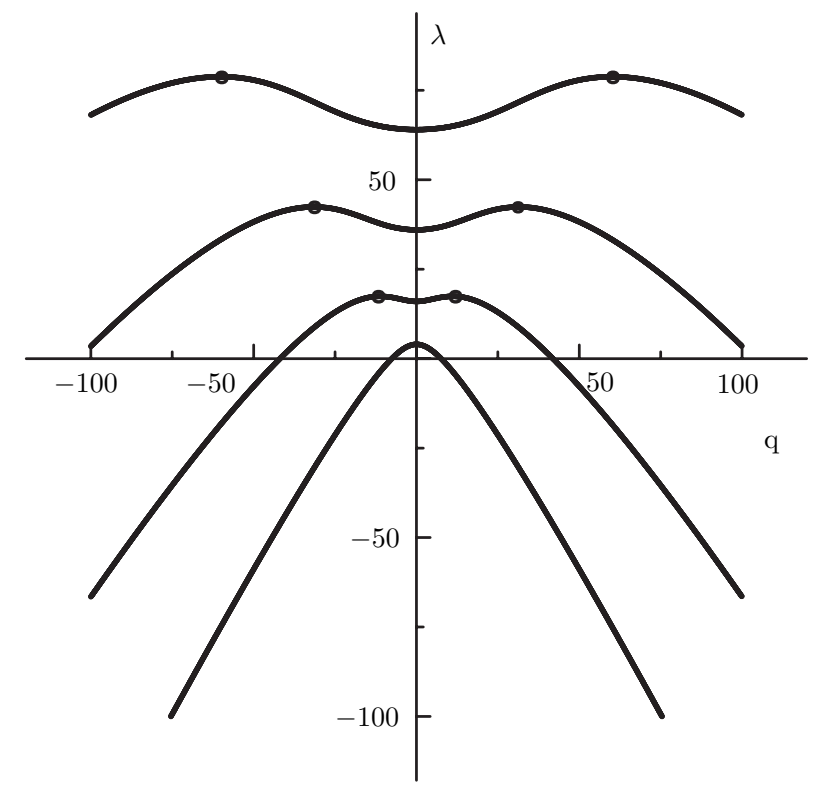

Figure 3. Real $q$ and real $\lambda$ curves $\left(\operatorname{se}_{2 m}(z, q)\right.$ type) 
TABLE 2. Intermediate results of iterations (with initial values) $(N=9$ and 10$)$

\begin{tabular}{|c|c|c|c||c|c|c|c|}
\hline$N$ & $n$ & $q_{n}$ & $\lambda_{n}$ & $N$ & $n$ & $q_{n}$ & $\lambda_{n}$ \\
\hline \hline 9 & 0 & 407.403706625 & 530.637818017 & 10 & 0 & 495.706449576 & 645.830622574 \\
& 1 & 409.880649886 & 533.270076226 & & 1 & 498.141239606 & 648.544906938 \\
& 2 & 409.673247606 & 533.746760069 & & 2 & 497.960506640 & 648.908929885 \\
& 3 & 409.674751506 & 533.744316297 & & 3 & 497.961367957 & 648.907648777 \\
& 4 & 409.674751715 & 533.744316177 & & 4 & 497.961368004 & 648.907648750 \\
\hline
\end{tabular}

where $\mathrm{d} \lambda / \mathrm{d} q=0$ holds (marked "•"). After Theorem 1 is applied, the following relation is obtained:

$$
\begin{aligned}
\left(\frac{\mathrm{d} \lambda}{\mathrm{d} q}\right)\left(\mathbf{y}^{T} \mathbf{y}\right) & =-\frac{2}{q}\left(\mathbf{x}^{T} \mathbf{x}\right), \\
\text { where } \mathbf{y} & =\left[B_{2}, B_{4}, B_{6}, \ldots\right]^{T} \in \ell^{2}, \\
\mathbf{x} & =\left[\sqrt{r_{4}} B_{2}, \sqrt{r_{8}} B_{8}, \sqrt{r_{12}} B_{12}, \ldots\right]^{T} \in \ell^{2} \quad\left(r_{n} \equiv n^{2}-\lambda\right) .
\end{aligned}
$$

The authors of [2, Chapter 3, (24b) and Figure 1] found, through experiments, that a group of $q-\lambda$ curves $\left\{a_{k}\right\}(k=1,2, \ldots)\left(a_{k}\right.$ corresponds to the $k$ th closest curve to the origin with $q$ and $\lambda$ real) have the following relation:

$$
a_{n}(h) \sim\left(\frac{n}{m}\right)^{2} a_{m}\left(\frac{m}{n} \cdot h\right),
$$

where $h^{2}=4 q$, and $m$ and $n$ are natural numbers. This of course is true of double pairs $(q, \lambda)$ too. Then, once a double pair is gained, one can utilize their values for the selection of a good initial candidate (which is often considered the key to the Newton method), with (4.3) for the other double pairs.

With such preparation done, the Newton method of two variables is executed. Table 2 tabulates the initial values $\left(q_{0}\right.$ and $\left.\lambda_{0}\right)$ and the values of $q_{n}$ and $\lambda_{n}$ for the 9 th and 10th double pairs closest to the origin. Only the first 12 digits are displayed (after rounding); $n$ represents the number of iterations. In each case (or $N=9,10$ ), two digits are already in agreement with the true pair $(q, \lambda)$ at the stage its initial value is determined. For the list of the first 30 double pairs, see [8].

4.3.2. Double pairs of type $\mathrm{d} \mu / \mathrm{d} \lambda=0$. In the previous case, the $q-\lambda$ graph was created for real $q$ and real $\lambda$. Next, let the new graph be plotted, this time for real

TABle 3. The first 10 double pairs $(q, \lambda)$ of type $\mathrm{d} \mu / \mathrm{d} \lambda=0$

\begin{tabular}{|c|c|c|}
\hline$N$ & $q$ & $\lambda$ \\
\hline \hline 1 & $i \cdot 6.928954758 \cdots$ & $11.19047359 \cdots$ \\
2 & $i \cdot 30.09677283 \cdots$ & $50.47501615 \cdots$ \\
3 & $i \cdot 69.59879327 \cdots$ & $117.8689241 \cdots$ \\
4 & $i \cdot 125.4354113 \cdots$ & $213.3725686 \cdots$ \\
5 & $i \cdot 197.6066786 \cdots$ & $336.9860439 \cdots$ \\
6 & $i \cdot 286.1126087 \cdots$ & $488.7093844 \cdots$ \\
7 & $i \cdot 390.9532062 \cdots$ & $668.5426056 \cdots$ \\
8 & $i \cdot 512.1284733 \cdots$ & $876.4857154 \cdots$ \\
9 & $i \cdot 649.6384116 \cdots$ & $1112.538718 \cdots$ \\
10 & $i \cdot 803.4830180 \cdots$ & $1376.701616 \cdots$ \\
\hline
\end{tabular}




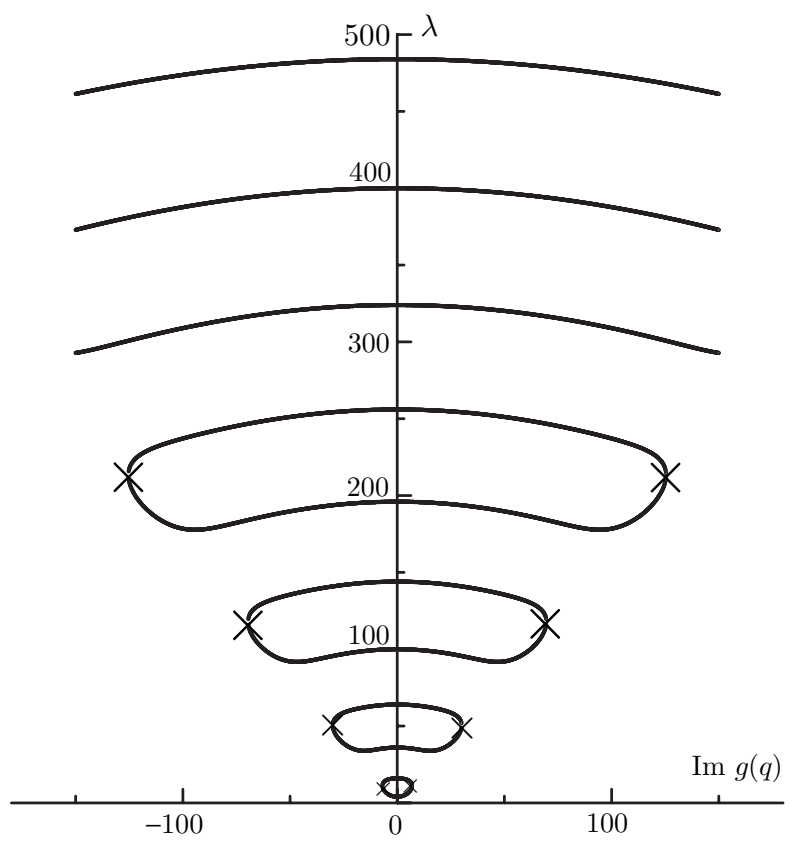

Figure 4 . Real $\lambda$ and purely imaginary $q$ curves $\left(s e_{2 m}(z, q)\right.$ type $)$

TABLE 4. Intermediate results of iterations (with initial values) $(N=5$ and 6$)$

\begin{tabular}{|c|c|c|c||c|c|c|c|}
\hline$N$ & $n$ & $q_{n}$ & $\lambda_{n}$ & $N$ & $n$ & $q_{n}$ & $\lambda_{n}$ \\
\hline \hline 5 & 0 & $i \cdot 195.992830179$ & 333.394638496 & 6 & 0 & $i \cdot 284.553617317$ & 485.259903288 \\
& 1 & $i \cdot 197.304187600$ & 335.345192031 & & 1 & $i \cdot 285.932050268$ & 487.572025430 \\
& 2 & $i \cdot 197.617867469$ & 336.745916724 & & 2 & $i \cdot 286.122018441$ & 488.630799983 \\
& 3 & $i \cdot 197.607725864$ & 336.984121128 & & 3 & $i \cdot 286.112706460$ & 488.709293001 \\
& 4 & $i \cdot 197.606678665$ & 336.986044253 & & 4 & $i \cdot 286.112608761$ & 488.709384477 \\
& 5 & $i \cdot 197.606678692$ & 336.986043950 & & 5 & $i \cdot 286.112608762$ & 488.709384476 \\
\hline
\end{tabular}

$\lambda$ and purely imaginary $q$ (in Figure 4). It is observed in Figure 4 that there should exist pairs of $\lambda$ (real) and $q$ (purely imaginary) such that $\mathrm{d} q / \mathrm{d} \lambda=0$, at the points marked " $\times$ ". As in the case of type $\mathrm{d} \lambda / \mathrm{d} \mu=0$, double pairs of the same accuracy were obtained. The first 10 double pairs are listed in Table 3.

It is naturally conjectured that (4.3) applies for real $\lambda$ and purely imaginary $q$ as well. The fact is that Table 3 was thus created. Table 4 describes the iterated values of $\left(q_{n}, \lambda_{n}\right)$ for the 5 th and 6 th closest double pairs. The notation of $n$ and $N$ are identical with Section 4.3 .1

\section{Concluding Remarks}

In this paper, a class of infinite matrices was set and theorems on their double pairs were shown. In addition to the theorems, a method was proposed for the computation of such double pairs by the Newton method of two variables. It was 
also found that the bisection method is useful when parameters are real. This method was applied to the zeros of $J_{\nu}(z)$ and $z J_{\nu}^{\prime}(z)+H J_{\nu}(z)$ and to the EVP of the Mathieu differential equation. These examples demonstrated that our method enables efficient and accurate computation of multiple zeros.

\section{REFERENCES}

[1] M. Abramowitz and I. A. Stegun, Handbook of Mathematical Functions, Dover, N.Y., 1972. MR 94b:00012

[2] Fayez A. Alhargan, A Complete Method for the Computations of Mathieu Characteristic Numbers of Integer Orders, SIAM Rev., 38 No.2 (1996), 239-255. MR 97h:33036

[3] T. M. Apostol, Mathematical Analysis (second edition), Addison-Wesley, 1974. MR 49:9123

[4] N. Asai, Y. Miyazaki, D. Cai, K. Hirasawa, and Y. Ikebe, Numerical Methods for $z J_{\nu}^{\prime}(z)+$ $H J_{\nu}(z)=0$ by Eigenvalue Problem, The Transactions of the Institute of Electronics, Information and Communication Engineers A, Vol. J79-A, No. 7 (1996), 1256-1265. (Later translated into English and appeared in Electronics and Communications in Japan, Part 3, Vol. 80, No. 7 (1997), 44-54.)

[5] W. Gautschi, Computational Aspects of Three-Term Recurrence Relations, SIAM Rev., 9 (1967), 24-82. MR 35:3927

[6] Y. Ikebe, N. Asai, Y. Miyazaki, and D. Cai, The Eigenvalue Problem for Infinite Complex Symmetric Tridiagonal Matrices with Application, Linear Algebra Appl., 241-243 (1996), 599-618. MR 97d:15016

[7] Y. Ikebe, Y. Kikuchi, I. Fujishiro, N. Asai, K. Takanashi, and M. Harada, The Eigenvalue Problem for Infinite Compact Complex Symmetric Matrices with Application to the Numerical Computation of Complex Zeros of $J_{0}(z)-i J_{1}(z)$ and of Bessel Functions $J_{m}(z)$ of Any Real Order m, Linear Algebra Appl., 194 (1993), 35-70. MR 94g:47025

[8] Y. Miyazaki, N. Asai, D. Cai, and Y. Ikebe, A Numerical Computation of the Inverse Characteristic Values of Mathieu's Equation, Transactions of the Japan Society for Industrial and Applied Mathematics, 8(2), (1998), 199-222 (in Japanese).

[9] Y. Miyazaki, N. Asai, D. Cai, and Y. Ikebe, The Computation of Eigenvalues of Spheroidal Differential Equations by Matrix Method, JSIAM Annual Meeting, (1997), 224-225 (in Japanese).

[10] Y. Miyazaki, Y. Kikuchi, D. Cai, and Y. Ikebe, Error Analysis for the Computation of Zeros of Regular Coulomb Wave Function and Its First Derivative, Math. Comp., 70 (2001), 11951204. MR 2001j:81271

[11] Y. Miyazaki, Y. Kikuchi, D. Cai, and Y. Ikebe, The Computation of Double Eigenvalues for Infinite Matrices of a Certain Class with Newton's Method, Abstracts of Plenary and Invited Lectures Delivered at the Second Congress ISAAC 1999 (1999), 148-149.

[12] G. N. Watson, A Treatise on the Theory of Bessel Functions, Cambridge Univ. Press, 1944. MR 6:64a

[13] J. Wimp, Computation with Recurrence Relation, Pitman Publishing, Boston, MA, 1984. MR 85f:65001

Faculty of Communications and Informatics, Shizuoka Sangyo University, Shizuoka 426-8668, JAPAN

E-mail address: yoshi@ssu.ac.jp

School of Computer Science and Engineering, University of Aizu, Fukushima-ken 965-8580, JAPAN

Faculty of Science, Division II, Tokyo University of Science, Tokyo, 162-8601, Japan

Institute of Information Sciences and Electronics, University of Tsukuba, Ibaraki 305-8573, JAPAN

Research Center for Information Science, Meisei University, Tokyo, 191-8506, Japan 